\title{
Genetic polymorphisms of cytochrome p4502E1 and susceptibility to alcoholic liver disease and hepatocellular carcinoma in a white population: a study and literature review, including meta-analysis
}

\author{
N A C S Wong, F Rae, K J Simpson, G D Murray, D J Harrison
}

\begin{abstract}
Aims - To investigate the associations between the Rsa I, Dra I, and Taq I genetic polymorphisms of cytochrome p4502E1 and susceptibility to alcoholic liver disease or to hepatocellular carcinoma.

Methods-DNA samples isolated from 61 patients with alcoholic liver disease, 46 patients with hepatocellular carcinoma, and 375 healthy controls were subjected to polymerase chain reaction amplification followed by digestion with the endonucleases Rsa I, Dra I, or Taq I. Meta-analysis was performed using data from previous studies of Rsa I polymorphism and the risk of alcoholic liver disease.

Results - No association was found between any of the three polymorphisms and susceptibility to hepatocellular carcinoma. The distributions of Rsa I and Dra I alleles among the patients with alcoholic liver disease were not significantly different from those among the control group. Meta-analysis of this data and previous data concerning Rsa I polymorphism and alcoholic liver disease risk failed to demonstrate any significant association between the two. However, the alcoholic liver disease group in this study showed a significantly lower frequency of the less common Taq I allele compared with the healthy control group (odds ratio, 0.33; $95 \%$ confidence interval, 0.12 to 0.78 ).

Conclusions-Possession of the less common Taq I cytochrome p4502E1 allele is associated with reduced susceptibility to alcoholic liver disease. There is no existing evidence that the Taq I polymorphism is directly associated with altered alcohol metabolism, but it might be in linkage disequilibrium with as yet unidentified protective factors.

(F Clin Pathol: Mol Pathol 2000;53:88-93)
\end{abstract}

Keywords: cytochrome p4502E1; alcoholic liver disease; hepatocellular carcinoma

Cytochrome p4502E1 (CYP2E1) is a key microsomal enzyme that metabolises alcohol in the non-alcohol dehydrogenase pathway. ${ }^{12}$ Because the gene encoding CYP2E1 shows several polymorphisms, it has been speculated that these polymorphisms might, at least in part, explain the inherited variability in suscep- tibility to alcoholic liver disease. ${ }^{13}$ To date, the groups that have searched for possible associations between CYP2E1 genetic polymorphisms and alcoholic liver disease susceptibility have reported varied, often contradictory, results. ${ }^{3-13}$ Some of these discrepancies might relate to CYP2E1 having different putative roles in the pathogenesis of alcoholic liver disease in different ethnic groups. ${ }^{7}$ There have been nine published reports of the relation between CYP2E1 polymorphisms and alcoholic liver disease in white populations. ${ }^{36-13}$ Rsa I/Pst I polymorphism was investigated in all nine studies, most of which were unable to demonstrate any significant association between the polymorphism and the risk of developing alcoholic liver disease. ${ }^{3-12}$ This conclusion has, however, not gained universal acceptance, particularly because one UK study reported a strong link between carriage of the less common Rsa I allele (c2) and an increased risk of developing alcoholic liver disease. ${ }^{8}$ The Dra I polymorphism of the CYP2E1 gene is considerably more prevalent in white populations than the Rsa I/Pst I polymorphism ${ }^{911}$ and, therefore, could conceivably play a more important role in inherited susceptibility for alcoholic liver disease. However, the four studies that have investigated this hypothesis produced conflicting results: the earliest study found the less common Dra I allele (C) to be less frequent in patients with alcoholic cirrhosis than in healthy controls ${ }^{3}$; two later studies reported the opposite, ${ }^{911}$ and the most recent study has shown no significant difference in $\mathrm{C}$ allele frequency between patients with alcoholic cirrhosis and healthy controls. ${ }^{12}$ The use of Taq I to demonstrate polymorphism of intron 7 of the CYP2E1 gene was first reported in $1987 .{ }^{14}$ Taq I polymorphism is as frequent in white populations as Dra I polymorphism, with the less common Taq I allele (A1) having a reported population frequency of 0.08 to $0.10{ }^{14-16}$ Therefore, it is surprising that there have been no reported studies into the relation between CYP2E1 Taq I polymorphism and susceptibility to alcoholic liver disease.

Because hepatic CYP2E1 has been shown to activate various carcinogens, there has been interest in whether certain CYP2E1 polymorphisms might predispose to liver cancer. ${ }^{17-19}$ We are aware of only one previous study that has investigated this hypothesis in a white population. ${ }^{19}$ Although Ladero and colleagues 
demonstrated that possession of the less common Rsa I/Pst I allele (c2) was associated with increased susceptibility for hepatocellular carcinoma, the relations between Dra I and Taq I polymorphisms and hepatocellular carcinoma risk were not studied.

Our study aims to investigate the above unresolved issues regarding CYP2E1 genetic polymorphisms and their relation to susceptibility for alcoholic liver disease and hepatocellular carcinoma. Hence, in a white population, we have: (1) performed meta-analysis of our data and data from previous studies of Rsa I/Pst I polymorphism and alcoholic liver disease risk to help reach a definitive conclusion about their relation; (2) retested the hypothesis that possession of the less common Dra I allele is associated with an increased risk of developing alcoholic liver disease; (3) studied the relation between Taq I polymorphism and susceptibility to alcoholic liver disease; and (4) investigated whether Dra I and/or Taq I polymorphisms are associated with susceptibility to hepatocellular carcinoma.

\section{Methods}

Blood samples were obtained from 61 patients with alcoholic liver disease receiving care at the Centre of Liver and Digestive Diseases at the Royal Infirmary of Edinburgh. These patients had a median age of 56 years (range, 32 to 90 ) and showed a male to female patient ratio of 2.2. All 61 patients had alcohol consumptions of greater than $60 \mathrm{~g} /$ day for greater than 10 years (median intake, $150 \mathrm{~g} /$ day for 18 years); these alcohol consumption data were obtained directly from the patients with, where possible, corroboration from relatives and/or partners. All 61 patients had undergone liver biopsies, which demonstrated at least one of the following features: alcoholic hepatitis, precirrhotic pericellular/perivenular fibrosis, and/or cirrhosis. None of the patients showed clinical, histological, or serological evidence of an alternative or additional cause of liver disease. Forty six consecutive hepatocellular carcinoma resection tissue sections (patient median age, 66 years; age range, 21 to 92 ; male to female patient ratio, 2.3) were obtained from the archives of the department of pathology, University of Edinburgh. The hepatocellular carcinoma group did not include any patients with clinical and/or histological evidence of alcoholic liver disease. Excluding such cases allowed us to study, in isolation, the relation between CYP2E1 polymorphisms and hepatocellular carcinoma risk, and to avoid the confounding effects of coexisting alcoholic liver disease. Three hundred and seventy five control blood samples were obtained from the Scottish National Blood Transfusion Service. The anonymous donors were aged between 18 and 65 years and consisted of roughly equal numbers of men and women. All 375 donors appeared healthy on routine questioning and examination, and met the usual criteria for blood donation. The use of healthy individuals as the control group as opposed to alcoholic patients with no clinical or biochemical evidence of alcoholic liver disease-as used by several previous studies ${ }^{6-9}$-is explained below. All patients and control individuals were white.

Because the study of patients with hepatocellular carcinoma represented only a sidearm of the main study, the number of patients with alcoholic liver disease required took precedence in the calculation of sample sizes. This calculation was performed using the computer software package Epi Info v6.04b (Centers for Disease Control and Prevention, Atlanta, USA) with the following variables: a power of $80 \%$; a significance level of $95 \%$; a control to patient ratio of $4: 1$; an expected proportion of control individuals possessing the less common allele of $7.0 \%$ for Rsa I, $19 \%$ for Dra I, and $25 \%$ for Taq I; and a significant difference defined as a threefold higher proportion of patients carrying the less common allele compared with control individuals. Based on the trends shown by previous studies on Rsa I and Dra I polymorphisms, we did not anticipate a decreased frequency of the less common alleles in the patient group. The expected proportions were derived from the genotyping of our control group, which had preceded our study. The largest sample sizes required were those required for Rsa I analysis, and consisted of 224 control individuals and 61 patients.

Our study design was approved by the Lothian Health Board ethical review committee, and informed consent was obtained from the patients attending the Centre of Liver and Digestive Diseases. DNA was obtained from sections of archival tissue samples without neoplastic changes or from fresh peripheral blood leucocytes, as has been described previously. ${ }^{20}{ }^{21}$

The endonucleases Rsa I and Pst I each detect a different polymorphism in the 5' flanking region of the CYP2E1 gene: an allele possessing a positive restriction point for Rsa I is designated as a c1 allele and one with a Pst I restriction point, a c2 allele. ${ }^{145}$ With rare exceptions, the two polymorphisms show complete linkage disequilibrium in white populations. ${ }^{6722}$ Therefore, for the purposes of our study, only Rsa I was used to demonstrate the polymorphisms, and absence of the Rsa I restriction point was taken to define a c2 allele.

Dra I digestion detects a polymorphism in intron 6 of the CYP2E1 gene and Taq I a polymorphism in intron $7 .{ }^{14} 16$ The more common and less common alleles demonstrated using Dra I are designated D and C, respectively, and those demonstrated using Taq I, A2 and A1, respectively. ${ }^{14} 16$

Polymerase chain reaction (PCR) for the Rsa I assay was carried out with an Omnigene thermocycler (Hybaid, Ashford, UK) with $200 \mu \mathrm{M}$ of each dNTP, 1.25 units of DNA polymerase (Advanced Biotechnologies, Epsom, UK), $50 \mathrm{ng}$ of each primer, and $1.5 \mathrm{mM}$ magnesium chloride. The primer sequences were 5'CCAGTCGAGTCTACATTGTCA and 5'TTCATTCTGTCTTCTAACTGG, as used in previous studies. ${ }^{45-9} 1123$ The amplification protocol comprised $94^{\circ} \mathrm{C}$ for 20 seconds, $54^{\circ} \mathrm{C}$ for 20 seconds, and $72^{\circ} \mathrm{C}$ for 20 seconds (36 cycles), followed by $54^{\circ} \mathrm{C}$ for 10 minutes, and $72^{\circ} \mathrm{C}$ for 10 minutes. PCR products were run 


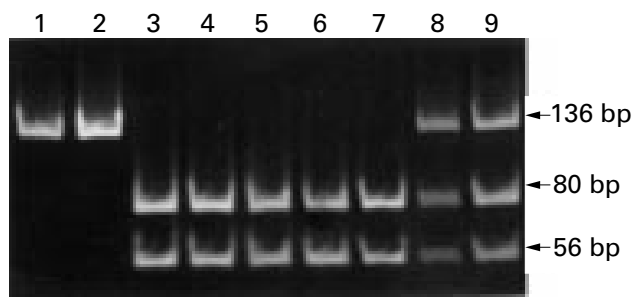

Figure 1 Electrophoresis of the PCR products of the CYP2E1 gene after digestion with the endonuclease Taq I. Lanes 1 and 2 indicate homozygosity for the less common allele (A1A1), lanes 3-7 homozygosity for the more common allele $(A 2 A 2)$, and lanes 8 and 9 heterozygosity (A2A1).

on a $2 \%$ agarose gel. The products were digested with Rsa I overnight at $37^{\circ} \mathrm{C}$, and the fragments separated on a $10 \%$ acrylamide gel.

PCR for the Dra I assay was carried out with an Omnigene thermocycler (Hybaid), with $200 \mu \mathrm{M}$ of each dNTP, 1.25 units of Taq polymerase (Life Technologies, Paisley, UK), TaqStart antibody (Clontech, Basingstoke, UK), $50 \mathrm{ng}$ of each primer, and $1.5 \mathrm{mM}$ magnesium chloride. The primer sequences were 5'-GACAGGGTTTCATCATGTTGG and 5'-AGTCGACATGTGATGGATCCA, as used in previous studies..$^{911} 24$ The amplification protocol comprised $94^{\circ} \mathrm{C}$ for 30 seconds, $62^{\circ} \mathrm{C}$ for 30 seconds, and $72^{\circ} \mathrm{C}$ for 30 seconds (36 cycles), followed by $62^{\circ} \mathrm{C}$ for two minutes, and $72^{\circ} \mathrm{C}$ for 10 minutes. PCR products were run on a $2 \%$ agarose gel. The products were digested with Dra I overnight at $37^{\circ} \mathrm{C}$, and the fragments separated on a $10 \%$ acrylamide gel.

PCR for the Taq I assay was carried out with an Omnigene thermocycler (Hybaid) with $200 \mu \mathrm{M}$ of each dNTP, 1.25 units of DNA polymerase (Advanced Biotechnologies), $50 \mathrm{ng}$ of each primer, and $1.5 \mathrm{mM}$ magnesium chloride. The primer sequences were 5'GGGCTTTCATCTTCATTTCGA and 5'CAAAATGTGGGCTTTCATCTG, and were designed from the CYP2E1 gene sequence published in the Genbank database. By introducing a single base mismatch, the upstream primer contains an engineered Taq I site as a control for the restriction enzyme. The amplification protocol comprised $94^{\circ} \mathrm{C}$ for 20 seconds, $56^{\circ} \mathrm{C}$ for 20 seconds, and $72^{\circ} \mathrm{C}$ for 15 seconds ( 36 cycles), followed by $56^{\circ} \mathrm{C}$ for two minutes, and $72^{\circ} \mathrm{C}$ for 10 minutes. PCR products were run on a $2 \%$ agarose gel. The products were digested with Taq I overnight at $65^{\circ} \mathrm{C}$, and the fragments run on an $8 \%$ acrylamide gel.

All gels were visualised by means of ethidium bromide staining and examined under ultraviolet irradiation.

Associations between disease groups and specific genotypes were analysed for significance by the $\chi^{2}$ test using the Yates correction or, where more appropriate, by the two tailed Fisher's exact test. Odds ratios (OR) and 95\% confidence intervals (CI) were calculated to assess relative risk of disease conferred by a particular allele. The above statistical analyses were carried out using the computer software package Epi Info v6.04b.

Data were extracted from eight of the nine previous studies of Rsa I/Pst I polymorphism and alcoholic liver disease in white populations for meta-analysis using both the "fixed" and the "random effects" models. Savolainen and colleagues' study could not be included in the analysis because of a lack of raw data about $\mathrm{c} 2$ allele frequency among its "healthy control" group. ${ }^{11}$ The decision to compare alcoholic liver disease groups with healthy control groups in the meta-analysis is explained below. Five of the eight studies included in the meta-analysis only investigated patients with cirrhosis 391012 whereas three, like our own, also included patients with alcoholic hepatitis in their alcoholic liver disease study groups. ${ }^{6813}$ Because neither of these two groups appeared more or less likely to demonstrate any association between c2 carriage rate and alcoholic liver disease risk, all eight studies were pooled and considered together in the meta-analysis. The meta-analysis was carried out using the computer software package Rev Man v3.1 (The Cochrane Collaboration, Copenhagen, Denmark).

\section{Results}

The PCR produced amplimers of the expected size, and differentiated between individual Rsa I (data not shown), Dra I (data not shown), and Taq I genotypes (fig 1) in the study groups. Among the hepatocellular carcinoma group,

Table 1 Distribution of Rsa I, Dra I, and Taq I genotypes and less common allele frequencies among patients with alcoholic liver disease (ALD), patients with hepatocellular carcinoma (HCC), and healthy controls

\begin{tabular}{|c|c|c|c|c|c|c|}
\hline & \multicolumn{3}{|c|}{ Number of individuals (\% of group) } & \multirow[b]{2}{*}{$\begin{array}{l}\text { Less } \\
\text { common } \\
\text { allele } \\
\text { frequency }\end{array}$} & \multirow[b]{2}{*}{$\begin{array}{l}\chi^{2} \text { value } \\
\text { ( } p \text { value })\end{array}$} & \multirow[b]{2}{*}{ Odds ratio $(95 \%$ CI } \\
\hline & $\begin{array}{l}\text { Homozygous } \\
\text { more common } \\
\text { allele }\end{array}$ & Heterozygous & $\begin{array}{l}\text { Homozygous less } \\
\text { common allele }\end{array}$ & & & \\
\hline Rsa I polymorphism & $c 1 c 1$ & $c 1 c 2$ & $c 2 c 2$ & & & \\
\hline Controls $(\mathrm{n}=375)$ & $350(93 \%)$ & $25(7 \%)$ & $0(0 \%)$ & 0.033 & - & 1.0 \\
\hline $\operatorname{ALD}(n=61)$ & $59(97 \%)$ & $2(3 \%)$ & $0(0 \%)$ & 0.016 & $(0.41) \dagger$ & $0.48(0.05$ to 1.98$)$ \\
\hline $\operatorname{HCC}(\mathrm{n}=28)$ & $27(96 \%)$ & $1(4 \%)$ & $0(0 \%)$ & 0.018 & $(\approx 1.00) \dagger$ & $0.53(0.01$ to 3.35$)$ \\
\hline Dra I polymorphism & $D D$ & $D C$ & $C C$ & & & \\
\hline Controls $(n=375)$ & $305(81 \%)$ & $68(18 \%)$ & $2(<1 \%)$ & 0.096 & - & 1.0 \\
\hline $\operatorname{ALD}(n=61)$ & $50(82 \%)$ & $11(18 \%)$ & $0(0 \%)$ & 0.090 & $<0.01(0.97)$ & $0.93(0.43$ to 1.84$)$ \\
\hline $\operatorname{HCC}(\mathrm{n}=31)$ & $24(77 \%)$ & $6(20 \%)$ & $1(3 \%)$ & 0.129 & $0.38(0.54)$ & $1.40(0.55$ to 3.10$)$ \\
\hline Taq I polymorphism & $A 2 A 2$ & $A 2 A 1$ & $A 1 A 1$ & & & \\
\hline Controls $(n=375)$ & $279(75 \%)$ & $91(24 \%)$ & $5(1 \%)$ & 0.135 & - & 1.0 \\
\hline $\operatorname{ALD}(\mathrm{n}=61)$ & $55(90 \%)$ & $6(10 \%)$ & $0(0 \%)$ & 0.049 & $6.35(0.01)$ & $0.33(0.12$ to 0.78$)$ \\
\hline $\operatorname{HCC}(n=44)$ & $36(82 \%)$ & $7(16 \%)$ & $1(2 \%)$ & 0.102 & $0.47(0.49)$ & $0.73(0.31$ to 1.52$)$ \\
\hline
\end{tabular}

Statistical calculations are based on comparisons of allele frequency.

tp Value using the two tailed Fisher's exact test.

CI, confidence interval. 
Table 2 Summary of the meta-analysis, using the "random effects model", of the current and previous studies of whether possession of the $c 2$ allele is related to the risk of developing alcoholic liver disease (ALD)

\begin{tabular}{lllll}
\hline Reference & $\begin{array}{l}\text { ALD/ } \\
\text { cirrhosis }\end{array}$ & $\begin{array}{l}\text { Healthy } \\
\text { controls }\end{array}$ & $\begin{array}{l}\text { Weight } \\
\text { (\%) }\end{array}$ & Odds ratio (95\% CI) \\
\hline Parsian et al $(1998)^{12}$ & $0 / 86$ & $2 / 178$ & 3.4 & $0.41(0.02$ to 8.59$)$ \\
Wong et al (current study) & $2 / 122$ & $25 / 750$ & 10.9 & $0.48(0.05$ to 1.98$)$ \\
Ingelman-Sundberg et al $(1993)^{3}$ & $3 / 112$ & $10 / 228$ & 12.3 & $0.60(0.16$ to 2.22$)$ \\
Agúndez et al $(1996)^{10}$ & $2 / 116$ & $7 / 274$ & 9.6 & $0.67(0.14,3.27)$ \\
Ball et al $(1995)^{7}$ & $3 / 74$ & $6 / 216$ & 11.2 & $1.48(0.36$ to 6.07$)$ \\
Lucas et al $(1996)^{9}$ & $9 / 220$ & $13 / 520$ & 18.7 & $1.66(0.70$ to 3.95$)$ \\
Grove et al $(1998)^{13}$ & $14 / 480$ & $4 / 242$ & 14.7 & $1.79(0.58$ to 5.49$)$ \\
Carr et al $(1995)^{6}$ & $5 / 106$ & $1 / 64$ & 6.0 & $3.12(0.36$ to 27.3$)$ \\
Pirmohamed et al $(1995)^{8}$ & $19 / 190$ & $3 / 200$ & 13.2 & $7.30(2.12$ to 25.1$)$ \\
Total & $57 / 1506$ & $71 / 2672$ & 100.0 & $1.41(0.78$ to 2.55$)$ \\
& & & &
\end{tabular}

^Number of c2 alleles/total number of alleles.

$\mathrm{CI}$, confidence interval.

reproducible diagnostic bands could not be produced in 18 samples for Rsa I, 15 samples for Dra I, and two samples for Taq I. The fact that the paraffin wax blocks for several of these samples were more than 10 years old suggests that the failure to yield diagnostic bands might be the result of age related deterioration of the DNA extracted from these samples.

Within the control group, the most even distribution of CYP2E1 genotypes was demonstrated using Taq I (less common allele frequency, 0.135), followed by Dra I (0.096), and then Rsa I (0.033).

There were no significant differences in the frequencies of the less common Rsa I allele between the control and alcoholic liver disease groups (table 1). Similarly, there were no significant differences in the frequencies of the less common Dra I allele between the control and alcoholic liver disease groups (table 1). In contrast, the frequency of the less common Taq I allele in the alcoholic liver disease group was significantly less than that in the control group $\left(\chi^{2}=6.35 ; \mathrm{p}=0.012 ;\right.$ table 1$)$.

There were no significant differences in the frequencies of the less common Rsa I, Dra I, or Taq I alleles between the hepatocellular carcinoma and control groups (table 1).

Comparisons of the observed distributions of Rsa I, Dra I, and Taq I genotypes and those predicted by allele frequencies by $\chi^{2}$ analysis showed that the populations studied were in Hardy-Weinberg equilibrium, indicating that the control and study groups were sufficiently random and representative (data not shown).

Meta-analysis using the fixed effects model demonstrated heterogeneity among the nine studies of c2 allele carriage rate and alcoholic liver disease risk (data not shown), therefore suggesting that analysis using the random effects model would be preferable. The latter yielded an overall OR of 1.41 (95\% CI, 0.78 to 2.55) and therefore failed to demonstrate any significant association between $\mathrm{c} 2$ carriage and susceptibility for alcoholic liver disease (table 2).

\section{Discussion}

Our study investigated the relation between different polymorphisms of the CYP2E1 gene and susceptibility to both alcoholic liver disease and hepatocellular carcinoma. Of the eight previous studies included in our meta-analysis, four compared the $\mathrm{c} 2$ carriage rate of patients with alcoholic liver disease with that of healthy individuals and also with that of alcoholic patients with no evidence of liver disease. ${ }^{6-9}$ Using the latter group as controls is theoretically preferable in permitting the study, in isolation, of the direct association between CYP2E1 genetic polymorphisms and risk of liver disease on a background of alcoholism. In the four mentioned studies, the "no liver disease alcoholic" groups were defined as alcoholic patients with either no clinical or no clinical or biochemical evidence of liver disease. However, in these groups, liver disease was not excluded histologically, even though almost a fifth of alcoholic patients without clinical or biochemical abnormalities may show advanced disease on liver biopsy. ${ }^{25}$ The subsequent potential for significant error in defining these "no liver disease alcoholic" groups discouraged us from performing meta-analysis to compare these groups with patients with alcoholic liver disease. For similar reasons, we had initially planned to use only alcoholic patients with no histological evidence of liver disease as our control group. However (as other investigators presumably found), we were unable to recruit large enough numbers of such individuals. The alcoholic liver disease/ cirrhosis patient groups used by previous studies were defined by documented alcohol consumption (range of median individual intake, 148-257 g/day for 10-17 years) and, in almost all cases, confirmatory histological features on liver biopsy. ${ }^{3}$ 6-10 1213 The "healthy" control groups used in these studies showed more heterogeneity, ranging from university staff ${ }^{6}$ to blood donors. ${ }^{7}$ Because alcohol intake was not recorded and liver biopsies were not performed in most of these control groups, it is theoretically possible that some of the groups may have inadvertently included a small number of patients with alcoholic liver disease. Another weakness of using healthy control groups is the possibility of recruiting individuals who have the genetic predisposition to develop alcoholic liver disease, but who have not drunk sufficient alcohol to develop the disease. Finally, although our control group was selected because of its similar ethnic background to our patient groups, like other case control association studies, our study may suffer from the "founder effect" or population admixture. These issues of selecting an appropriate control group can be circumvented through the use of family studies, although the recruitment of relatives for such analyses does itself introduce new logistical problems. ${ }^{26}$

Accepting the aforementioned limitations, in keeping with the individual findings of most previous studies on the subject, we found no significant association between Rsa I/Pst I polymorphism and susceptibility to alcoholic liver disease in our own study population or from our meta-analysis (tables 2 and 3). It is difficult to reconcile this absence of association with the recent findings of Pirmohamed and colleagues, who used similar methodologies to our study and previous studies, but found possession of the less common allele (c2) to be strongly associated with an increased risk of developing alcoholic liver disease. ${ }^{8}$ However, 
Table 3 Summary of the findings of previous studies on the association between CYP2E1 genetic polymorphisms and susceptibility to alcoholic liver disease (ALD) in white populations

\begin{tabular}{|c|c|c|c|c|c|c|}
\hline Reference & Population studied & Study group (n) & $\begin{array}{l}\text { c2 allele } \\
\text { frequency }\end{array}$ & $p$ Value & $\begin{array}{l}\text { C allele } \\
\text { frequency }\end{array}$ & $p$ Value \\
\hline Ingelman-Sundberg et al (1993) & Italian & $\begin{array}{l}\text { Control\# (114) } \\
\text { Cirrhosis }\end{array}$ & $\begin{array}{l}0.044 \\
0.027\end{array}$ & $0.56 \$$ & $\begin{array}{l}0.083 \\
0.036\end{array}$ & $0.22^{\star}$ \\
\hline Carr et al $(1995)^{6}$ & North American & $\begin{array}{l}\text { Control\# (32) } \\
\text { ALD (53) }\end{array}$ & $\begin{array}{l}0.016 \\
0.047\end{array}$ & $0.41 \S$ & - & - \\
\hline Ball et al $(1995)^{7}$ & British & $\begin{array}{l}\text { Control\# (114) } \\
\text { Cirrhosis } \mid(37)\end{array}$ & $\begin{array}{l}0.028 \\
0.041\end{array}$ & $0.70 \$$ & - & - \\
\hline Pirmohamed et al $(1995)^{8}$ & British & $\begin{array}{l}\text { Control\# (100) } \\
\text { ALD (95) }\end{array}$ & $\begin{array}{l}0.015 \\
0.100\end{array}$ & $0.0006^{\star}$ & - & - \\
\hline Lucas et al $(1996)^{9}$ & French & $\begin{array}{l}\text { Control\# (260) } \\
\text { Cirrhosis } \mid(110)\end{array}$ & $\begin{array}{l}0.025 \\
0.041\end{array}$ & $0.35^{\star}$ & $\begin{array}{l}0.079 \\
0.146\end{array}$ & $0.008^{\star}$ \\
\hline Agúndez et al $(1996)^{10}$ & Spanish & $\begin{array}{l}\text { Control\# (137) } \\
\text { Cirrhosis }\end{array}$ & $\begin{array}{l}0.026 \\
0.017\end{array}$ & $\approx 1.00 \S$ & - & - \\
\hline Savolainen $(1997)^{11}$ & Finnish & $\begin{array}{l}\text { No ALD (33) } \\
\text { ALD (207) }\end{array}$ & $\begin{array}{l}t \\
0.010\end{array}$ & - & $\begin{array}{l}0.045 \\
0.130\end{array}$ & $0.08^{\star}$ \\
\hline Parsian et al $(1998)^{12}$ & North American & $\begin{array}{l}\text { Control\# (89) } \\
\text { Cirrhosis } \$(43)\end{array}$ & $\begin{array}{l}0.011 \\
0.000\end{array}$ & $\approx 1.00 \$$ & $\begin{array}{l}0.107 \\
0.093\end{array}$ & $0.90^{\star}$ \\
\hline Grove et al $(1998)^{13}$ & British & $\begin{array}{l}\text { Control\# (121) } \\
\text { ALD }(240)\end{array}$ & $\begin{array}{l}0.017 \\
0.029\end{array}$ & $0.44^{\star}$ & $\begin{array}{l}- \\
-\end{array}$ & - \\
\hline Wong et al (current study) & British & $\begin{array}{l}\text { Control\# (375) } \\
\text { ALD (61) }\end{array}$ & $\begin{array}{l}0.033 \\
0.016\end{array}$ & $0.41 \S$ & $\begin{array}{l}0.096 \\
0.090\end{array}$ & $0.97^{\star}$ \\
\hline
\end{tabular}

${ }^{\star}$ Chi squared test with Yates correction; \two tailed Fisher's exact test; \#healthy controls; $₫$ alcohol related cirrhosis; †no raw data given.

regardless of the reasons for this discrepancy, the low frequencies at which the $\mathrm{c} 2$ allele exists in white populations ${ }^{3-13162227}$ suggests that, if present, it only makes a minor contribution to inherited susceptibility to alcoholic liver disease.

The less common Dra I allele (C) is found two to four times more frequently in healthy white control groups than the c2 allele. ${ }^{3} 9111627$ To date, two research groups have demonstrated the frequency of the $\mathrm{C}$ allele to be higher among patients with alcoholic liver disease than among healthy controls, ${ }^{9}$ or alcohol drinkers without liver disease. ${ }^{11}$ Although an earlier study reported the opposite, ${ }^{3}$ the association was not significant. In agreement with the findings of Parsian and colleagues' recent study, ${ }^{12}$ our data failed to show a significant association between possession of the less common Dra I allele and susceptibility to alcoholic liver disease. It is possible, however, that our findings and those of Parsian and colleagues represent a type II error, because our alcoholic liver disease group and their alcoholic cirrhotic group were smaller than those analysed in the French and Finnish studies (table 3).

We found possession of the less common Taq I allele (A1) to be associated with a lower risk of developing alcoholic liver disease. A literature search failed to reveal any previous work on Taq I polymorphism of the CYP2E1 gene and alcoholic liver disease susceptibility. CYP2E1 activity is induced by chronic alcohol consumption and the enzyme has been implicated in the pathogenesis of alcoholic liver disease through the generation of reactive oxygen species and the promotion of lipid peroxidation. ${ }^{1}$ The relation between CYP2E1 genotype and phenotype is controversial. Although earlier in vitro work suggested that the c2 allele was associated with increased inducibility of the enzyme, ${ }^{23}$ several recent studies have failed to demonstrate such an association in vivo. ${ }^{1622}$ To date, there has only been one study relating Taq I polymorphism to hepatic CYP2E1 activity. ${ }^{16}$ Because Carrière and colleagues found no association between the two, it is possible that our findings regarding the A1 allele are attributable to its linkage to some as yet unidentified protective factor(s).

In contrast to the findings of a recent Spanish study, ${ }^{19}$ we were unable to demonstrate any association between possession of the c 2 allele and increased risk of developing hepatocellular carcinoma. Interestingly, Ladero and colleagues only found a significant association among patients with hepatocellular carcinoma who had high daily alcohol intakes, ${ }^{19}$ a group excluded from our analysis. The arguments raised earlier in the discussion point against this association being caused by $\mathrm{c} 2$ allele carriage increasing susceptibility to alcoholic cirrhosis and, thus, indirectly increasing susceptibility to hepatocellular carcinoma. Indeed, the same workers failed to relate Rsa I/Pst I polymorphism to alcoholic liver disease risk in a later study. ${ }^{10}$ It is not certain whether alcohol or its metabolites have a direct hepatocarcinogenic effect ${ }^{28}$ or, as discussed above, whether CYP2E1 activity and inducibility varies with different CYP2E1 genotypes. Thus, confirming and explaining the discrepant results of our study and the Spanish study will require further analysis of c2 allele carriage among alcoholic and non-alcoholic patients with hepatocellular carcinoma in other white populations.

In conclusion, we were unable to demonstrate any association between three polymorphisms of the CYP2E1 gene and susceptibility to hepatocellular carcinoma, or between Rsa I or Dra I polymorphism and susceptibility to alcoholic liver disease. Meta-analysis of previous studies and our own data failed to show any significant relation between carriage of the $\mathrm{c} 2$ allele and alcoholic liver disease risk. However, the findings of this first reported study of Taq I polymorphism and liver disease suggest possession of the A1 allele reflects a decreased risk of developing alcoholic liver disease. Further work is required to determine whether such an association exists in other white populations and other ethnic groups and, if so, to 
characterise the mechanisms that underlie the association.

This study was funded by a grant from the Scottish Office. Data from this study were presented at the 177 th meeting of the
Pathological Society of Great Britain and Ireland, held at the Pathological Society of Great Britain and
University of Leicester, 1-3 July 1998, UK.

1 Song BJ, Koop DR, Cederbaum AI, et al. Ethanol-induced cytochrome p450 (CYP2E1): biochemistry, molecula cytochrome p450 (CYP2E1): biochemistry, molecular
biology and clinical relevance: 1996 update. Alcohol Clin biology and clinical relevance:
Exp Res 1996;20:138A-46A.

2 Leiber CS. Ethanol metabolism, cirrhosis and alcoholism. Clin Chim Acta 1997;257:59-84.

3 Ingelman-Sundberg M, Johansson I, Yin $\mathrm{H}$, et al. Ethanolinducible cytochrome p4502E1: genetic polymorphism, regulation, and possible role in the aetiology of alcoholinduced liver disease. Alcohol 1993;10:447-52.

4 Maezawa Y, Yamauchi M, Toda G. Association between restriction fragment length polymorphism of the human cytochrome p450IIEI gene and susceptibility to alcoholic liver cirrhosis. Am 7 Gastroenterol 1994;89:561-5.

5 Tsutsumi M, Takada A, Wang J-S. Genetic polymorphisms of cytochrome p4502E1 related to the development of alcoholic liver disease. Gastroenterology 1994;107:1430-5.

6 Carr LG, Hartleroad JY, Liang Y, et al. Polymorphism at the P450IIEI locus is not associated with alcoholic liver disease in Caucasian men. Alcohol Clin Exp Res 1995;19:182-4.

7 in Caucasian men. Alcohol Clin Exp Res 1995;19:182-4. the c2 allele at the cytochrome p450IIEI gene and alcohol the c2 allele at the cytochrome p450IIEI gene and alcohol
induced liver disease, alcohol Korsakoff's syndrome or induced liver disease, alcohol Korsakoff's syndrome or alcohol deper

8 Pirmohamed M, Kitteringham NR, Quest LJ, et al. Genetic polymorphism of cytochrome p4502E1 and risk of alcoholic liver disease in Caucasians. Pharmacogenetic 1995;5:351-7.

9 Lucas D, Ménez C, Floch F, et al. Cytochrome p4502E1 and p4501A1 genotypes and susceptibility to cirrhosis or upper aerodigestive tract cancer in alcoholic Caucasians. Alcohol Clin Exp Res 1996;20:1033-7.

10 Agúndez J, Ladero J, Díaz-Rubio $M$, et al. Rsa I polymorphism at the cytochrome p4502E1 locus is not related to the risk of alcohol-related severe liver disease. Liver 1996;16:380-3.

11 Savolainen VT, Pajarinen J, Perola M, et al. Polymorphism in the cytochrome p4502E 1 gene and risk of alcoholic liver in the cytochrome p4502E1 gen

12 Parsian A, Cloninger R, Zhang ZH. Association studies of polymorphisms of CYP2E1 gene in alcoholics with cirrhosis, antisocial personality, and normal controls. Alcohol Clin Exp Res 1998;22:888-91.

13 Grove J, Brown AStJM, Daly AK, et al. The RsaI polymorphism of CYP2E1 and susceptibility to alcoholic liver dis- ease in Caucasians: effect on age of presentation and dependence on alcohol dehydrogenase genotype. Pharmacogenetics 1998;8:335-42.

14 McBride OW, Umeno M, Gelboin HV, et al. A Taq I polymorphism in the human P450IIEI gene on chromosome 10 (CYP2E). Nucleic Acids Res 1987;15:10071.

15 Persson I, Johansson I, Bergling $\mathrm{H}$, et al. Genetic polymorphisms of cytochrome p4502E1 in a Swedish population. Relationship to incidence of lung cancer. FEBS Lett 1993;319:207-11.

16 Carrière V, Berthou F, Baird S, et al. Human cytochrome p4502E1 (CYP2E1): from genotype to phenotype. Pharmacogenetics 1996;6:203-11.

17 Chen C-J, Yu M-W, Liaw Y-F. Epidemiological characteristics and risk factors of hepatocellular carcinoma. $\mathcal{F}$ Gastroenterol Hepatol 1997;12:S294-308.

18 Yu M-W, Gladek-Yarborough A, Chiamprasert S, et al. Cytochrome p4502E1 and glutathione S-transferase M1 polymorphisms and susceptibility to hepatocellular carcinoma. Gastroenterology 1995;109:1266-73.

19 Ladero JM, Agúndez JAG, Rodríguez-Lescure A, et al. Rsa I polymorphism at the cytochrome p4502E1 locus and risk of hepatocellular carcinoma. Gut 1996;39:330-3

20 Smith CAD, Gough AC, Leigh PN, et al. Debrisoquine hydroxylase gene polymorphisms and susceptibility to Parkinson's disease. Lancet 1992;339:1375-7.

21 Cantlay AM, Lamb D, Gillooly $\mathrm{M}$, et al. Association between the CYP1A1 gene polymorphism and susceptibility to emphysema and lung cancer. F Clin Pathol: Mol Pathol 1995;48:M210-14.

22 Kim RB, O'Shea D, Wilkinson GR. Interindividual variability of chlorzoxazone 6-hydroxylation in men and women and its relationship to CYP2E1 genetic polymorphisms. Clin Pharmacol Ther 1995;57:645-55.

23 Hayashi S, Watanabe J, Kawajiri K. Genetic polymorphisms in the 5 ' flanking region change transcriptional regulation of the human cytochrome p450 IIEI gene. F Biochem 1991; 110:559-65.

24 Hirvonen A, Husgafvel-Pursiainen K, Anttila S, et al. The human CYP2E1 gene and lung cancer: Dra I and Rsa I restriction length polymorphisms in a Finnish study population. Carcinogenesis 1993:14:85-8.

25 Bruguera M, Bordas JM, Rodes J. Asymptomatic liver disease in alcoholics. Arch Pathol Lab Med 1977;101:644-7.

26 Bassendine MF, Day CP. The inheritance of alcoholic liver disease. Ballieres Clin Gastroenterol 1998;12:317-35.

27 Stephens EA, Taylor JA, Kaplan N, et al. Ethnic variation in the CYP2E1 gene: polymorphism analysis of 695 AfricanAmericans, European-Americans and Taiwanese. Pharmacogenetics 1994;4:185-92.

28 Anthony PP. Tumours and tumour-like lesions of the liver and biliary tract. In: MacSween RNM, Anthony PP, Scheuer PJ, et al, eds. Pathology of the liver, 3rd ed. Edinburgh: Churchill Livingstone, 1994:635-711. 